\title{
A wider audience for Japanese research through use of the Web
}

\section{Dear Editor:}

As one of the international guest speakers at the recent meeting of the Japanese Gastric Cancer Association, I wish to make some observations which might be of wider interest. Firstly I have to say that it was a great privilege to be able to attend this meeting and to be brought up-to-date with the latest Japanese research. The provision of simultaneous translation on the first day of the meeting was an essential factor in facilitating communication.

While it is undoubtedly the case that an increasing amount of Japanese research output is appearing in major international journals, I am aware that much first-class work still does not reach an international audience. I think this particularly applies to clinically based research rather than experimental work or basic science. Those of us who are more familiar with studies in Japan endeavor to restore the balance by citations in our writing or by reference to such work in oral communications, but this has a limited effect. More is achieved through greater participation in international meetings and, in my own field, the attendance of Japanese pathologists at a recent meeting of the Pathological Society of Great Britain provided a muchvalued contribution. Nevertheless I feel that more could be done to ensure greater "penetration" of your findings into Western research and practice.

I wonder if more use could be made of the Web? Could free access be provided to the English abstracts (and possibly illustrations) from your journals? Journals like Gastric Cancer and Stomach and Intestine have a very limited number of personal subscribers outside Japan. Access via the Web would greatly increase readership and dissemination of information. There are, of course, important financial considerations, but this is an important medium of medical publishing which would increase Japanese-Western communication and be to our mutual benefit.

\author{
Michael F. Dixon \\ Professor of Gastrointestinal Pathology \\ Division of Pathological Sciences \\ University of Leeds \\ Leeds LS2 9JT, UK
}

\section{Editor's reply}

As one of the Editors-in-Chief, I appreciate Prof. Dixon's participation at the 72nd Congress of the Japanese Gastric Cancer Association (JGCA) held at Niigata (see Meeting Report, page 1 in this issue) and the suggestions in his letter to us. Since Gastric Cancer is the official journal of JGCA, "greater penetration" of Japanese findings into world research and practice is one of its primary goals. I am delighted to announce that the journal contents and abstracts are now available on the Web via the Springer-Verlag site (http://link.springer.de). Unfortunately, the full text and illustrations of papers currently are available only to those with passwords given to journal subscribers or "subscribing members" of the associations.

It has recently been debated how much we should provide free of charge on the website, and this is the situation with many similar international medical journals. The widely available World Wide Web is attractive in terms of the free passage of both Japanese and Western literature on the international stage, if the obvious financial difficulties can be overcome or a lead taken by journals with high impact factor. Prof. Dixon's suggestion will, however, be seriously considered in our future discussion.

Oichiro Kobori

Editor-in-Chief, Gastric Cancer 